\title{
Theoretical Analysis of High-Harmonic Generation in Solids
}

\author{
G. Vampa, ${ }^{1, *}$ C. R. McDonald, ${ }^{1, \dagger}$ G. Orlando, ${ }^{1}$ D. D. Klug, ${ }^{2}$ P. B. Corkum, ${ }^{1,2}$ and T. Brabec ${ }^{1}$ \\ ${ }^{1}$ Department of Physics, University of Ottawa, Ottawa, Ontario K1N 6N5, Canada \\ ${ }^{2}$ National Research Council of Canada, Ottawa, Ontario K1A OR6, Canada
}

(Received 9 August 2013; published 13 August 2014)

\begin{abstract}
We investigate theoretically high-harmonic generation (HHG) in bulk crystals exposed to intense midinfrared lasers with photon energies smaller than the band gap. The two main mechanisms, interband and intraband HHG, are explored. Our analysis indicates that the interband current neglected so far is the dominant mechanism for HHG. Saddle point analysis in the Keldysh limit yields an intuitive picture of interband HHG in solids similar to atomic HHG. Interband and intraband HHG exhibit a fundamentally different wavelength dependence. This signature can be used to experimentally distinguish between the two mechanisms in order to verify their importance.
\end{abstract}

DOI: 10.1103/PhysRevLett.113.073901

PACS numbers: 42.65.Ky, 42.50.Hz, 72.20.Ht, 78.47.-p

The interaction of intense laser fields with matter resulting in nonperturbative nonlinear dynamics has been extensively studied in atomic and molecular gases over the last two decades, revealing many new effects [1]. The most prominent of these processes is high-harmonic generation (HHG), which has resulted in the birth of attosecond science [2] and in new ultrafast imaging methods, such as molecular tomography [3] and spectroscopy [4]. Less attention has been paid to corresponding processes in the condensed matter phase, where the focus has been on laser micromachining and material modification $[5,6]$. Recent experiments have demonstrated that intense laser solid interactions offer a wide range of other phenomena and applications to be explored [7-9], including HHG in solids [10-13].

The goal of this Letter is to understand the main mechanism driving HHG in solids in the underresonant limit, with midinfrared (mid-ir) laser frequencies smaller than the band gap energy. HHG in solids can originate from two channels, an intraband current arising from nonlinear currents in the individual bands and an interband current due to polarization buildup between the valence and conduction band [14]. The question regarding which of the two processes is dominant has not yet been settled.

Theoretically, HHG in solids has been investigated in the single active electron approximation for 1D and 2D twoband models, either by numerical $[14,15]$ or analytical approaches [16-18]. The influence of propagation effects has also been investigated recently [18]. In the resonant limit the full single electron, 1D, two-band, density matrix equations have been solved numerically and both HHG currents have been investigated [14]. All previous work on HHG in the underresonant limit has not relied on a full solution of the two band equations, and has exclusively investigated the intraband current [15-18]; the interband current has not been considered yet.

Our analysis is based on a solution of the threedimensional (3D) density matrix equations for a two-band single active electron model similar to Ref. [14]. We solve the full two-band density matrix equations numerically; interband and intraband HHG are considered in the wavelength range made accessible by the latest generation of mid-ir high power lasers $(2-6 \mu \mathrm{m})$. We also show how to solve the two band equations analytically by generalizing the Lewenstein approach for atomic HHG $[19,20]$ to the density matrix formalism and to HHG in solids. Our analysis reveals the following main insights.

(i) Numerical results suggest interband HHG to be the dominant channel over most of the investigated mid-ir range, as long as the emitted harmonic frequency is above the band gap. Saddle point integration in the KeldyshLewenstein limit reveals the following mechanism responsible for interband HHG: after the creation of an electron-hole pair by tunnel ionization, electrons and holes are accelerated and driven apart by the laser field. When an electron and a hole reencounter each other, recombination can occur resulting in the emission of a harmonic photon; the wave vector at the time of recombination determines the energy difference (gap) between the electron-hole pair and therewith the emitted photon energy. By comparison, in atoms an electron is ionized and accelerated by the laser field and ultimately driven back to the core, which is stationary due to its weight. Upon recombination it releases its kinetic energy as a high energy photon.

(ii) In the absence of dephasing, higher order returns create a noisy continuumlike structure, which masks the odd harmonic spectrum. Dephasing times around an optical half-cycle ( $\sim 5 \mathrm{fs})$ are necessary to suppress higher returns and to obtain agreement with the clean odd harmonic structure observed in experiments $[10,13]$. This opens a novel way for determining dephasing times without the need of ultrashort laser sources.

(iii) For $\lambda>4 \mu \mathrm{m}$ the wavelength dependence of intraband and interband currents exhibits different signs, which 
presents a strong signature allowing us to experimentally settle the importance of the two contributions.

Our analysis of $\mathrm{HHG}$ in $\mathrm{ZnO}$ [10] is based on a 3D, two-band, tight-binding model coupled to a laser field $\mathbf{F}(t)=\hat{\mathbf{x}} F_{0} f(t)$; the dipole approximation is used, as the laser wave vector $(\mathbf{k} \| \hat{\mathbf{z}})$ is much smaller than the reciprocal lattice vectors. Further, atomic units are used throughout the Letter, unless stated otherwise. The laser is characterized by field amplitude $f(t)$, consisting of a temporal Gaussian envelope with a FWHM equal to 10 cycles and cosine carrier with frequency $\omega_{0}$.

The orientation of the reciprocal lattice of $\mathrm{ZnO}$ (wurtzite structure) is chosen so that $\hat{\mathbf{x}}\|\Gamma-M, \hat{\mathbf{y}}\| \Gamma-K$, and $\hat{\mathbf{z}} \| \Gamma-$ $A$ (optical axis). The valence band (holes), conduction band (electrons), and band gap are determined by $E_{v}=-\Delta E_{v}$, $E_{c}=E_{\mathrm{g}}+\Delta E_{c}$, and $\varepsilon_{g}=E_{c}-E_{v}=E_{\mathrm{g}}+\Delta E_{\mathrm{g}}$, respectively. We chose the valence band with the lightest mass along the laser polarization direction yielding the largest contribution to tunnel ionization. As there is some uncertainty in the band parameters, as obtained from various experimental and theoretical approaches, we have investigated HHG with bands obtained from two different methods in Ref. [21]: density functional theory in the local density approximation (LDA-ABINIT) and the nonlocal empirical pseudopotential method (NL-EPM). All conclusions drawn from our analysis are confirmed by both band models. Here, the results for the LDA-ABINIT are shown. The complete $3 \mathrm{D}$ band in the first Brillouin zone (BZ) is approximated as the sum over the three 1D bands.

Intense laser solid interaction is modeled using the two level density matrix equations [22]:

$$
\begin{aligned}
\dot{\pi}(\mathbf{K}, t) & =-\frac{\pi(\mathbf{K}, t)}{T_{2}}-i \Omega(\mathbf{K}, t) w(\mathbf{K}, t) e^{-i S(\mathbf{K}, t)}, \\
\dot{n}_{m}(\mathbf{K}, t) & =i s_{m} \Omega^{*}(\mathbf{K}, t) \pi(\mathbf{K}, t) e^{i S(\mathbf{K}, t)}+\text { c.c. }
\end{aligned}
$$

where $n_{m}$ is the the valence $(m=v)$ and conduction ( $m=c$ ) band population, and $w=n_{v}-n_{c}$ is the population difference. Initially all electrons are in the valence band; $s_{m}=-1,1$ for $m=v, c$, respectively. The crystal momentum $\mathbf{k}$ has been transformed into a frame moving with the vector potential $-d \mathbf{A} / d t=\mathbf{F}, \mathbf{K}=\mathbf{k}-\mathbf{A}(t)$. As a result, the first Brillouin zone is also shifted to $\overline{\mathrm{BZ}}=\mathrm{BZ}-\mathbf{A}(t)$. Further, $T_{2}$ accounts for dephasing, $S(\mathbf{K}, t)=\int_{-\infty}^{t} \varepsilon_{\mathrm{g}}\left[\mathbf{K}+\mathbf{A}\left(t^{\prime}\right)\right] d t^{\prime}$ is the classical action, $\Omega(\mathbf{K}, t)=\mathbf{F}(t) \mathbf{d}[\mathbf{K}+\mathbf{A}(t)]$ is the Rabi frequency, and $\mathbf{d}(\mathbf{k})=i \int d^{3} \mathbf{x} u^{*}{ }_{\mathrm{v}, \mathbf{k}}(\mathbf{x}) \nabla_{\mathbf{k}} u_{\mathrm{c}, \mathbf{k}}(\mathbf{x})$ is the transition dipole moment, with $u_{\mathrm{m}, \mathbf{k}}$ the periodic part of the Bloch function. The numerical value of the dipole moment at the $\Gamma$ point, $\mathbf{d}=(3.46,3.46,3.94)$, has been extracted from tabulated data [22], and its $k$ dependence is neglected in our analysis. Finally $\pi$ determines the polarization by

$$
\mathbf{p}(\mathbf{K}, t)=\mathbf{d}[\mathbf{K}+\mathbf{A}(t)] \pi(\mathbf{K}, t) e^{i S(\mathbf{K}, t)}+\text { c.c.. }
$$

For the integration of Eqs. (1) for linearly polarized pulses we used 600 points (full BZ) along $\Gamma-M$ and 200 points (half BZ) along each of the other two directions. Computation time for a laser wavelength $\lambda=3.25 \mu \mathrm{m}$ on an Intel Xeon E5 $(2.3 \mathrm{GHz})$ is 2.5 days.

The above equations are identical to the two-band semiconductor equations [31] in the single electron limit. The main difference is the frame transformation, which allows more efficient numerical integration, as it removes differentiation $\nabla_{\mathbf{k}}$ from the standard two-band equations; it also allows generalization of the analytical tools developed for atomic HHG [19] to solids. A derivation and the connection between the Schrödinger $[19,20]$ and the density matrix approach is given in the Supplemental Material [22].

Finally, HHG in solids is determined by an intraband $\mathbf{j}_{\text {ra }}$ and interband $\mathbf{j}_{\mathrm{er}}$ contribution, which are, respectively, given by $[14,31]$

$$
\begin{aligned}
& \mathbf{j}_{\mathrm{ra}}(t)=\sum_{m=c, v} \int_{\overline{\mathrm{BZ}}} \mathbf{v}_{m}[\mathbf{K}+\mathbf{A}(t)] n_{m}(\mathbf{K}, t) d^{3} \mathbf{K}, \\
& \mathbf{j}_{\mathrm{er}}(t)=\frac{d}{d t} \int_{\overline{\mathrm{BZ}}} \mathbf{p}(\mathbf{K}, t) d^{3} \mathbf{K},
\end{aligned}
$$

where the band velocity is defined by $\mathbf{v}_{m}(\mathbf{k})=\nabla_{\mathbf{k}} E_{m}(\mathbf{k})$. The high-harmonic spectrum is obtained from the Fourier transform (FT) of $\mathbf{j}_{\mathrm{t}}=\mathbf{j}_{\mathrm{ra}}+\mathbf{j}_{\mathrm{er}}$, as $\left|\mathrm{FT}\left\{\mathbf{j}_{\mathrm{t}}\right\}\right|^{2}$.

In order to better understand the physical processes driving intraband and interband HHG in solids, it is useful to explore Eqs. (1) by using the Keldysh approximation [20] $w(t) \approx 1$ in Eq. (1a). This decouples Eqs. (1) so that they can be formally integrated. Inserting the result into Eqs. (3), we find

$$
\begin{aligned}
\mathbf{j}_{\mathrm{ra}}(\omega)= & \sum_{m=c, v} s_{m} \int_{\mathrm{BZ}} d^{3} \mathbf{k} \mathbf{v}_{m}(\mathbf{k}) \\
& \times \int_{-\infty}^{\infty} d t e^{-i \omega t} \int_{-\infty}^{t} d t^{\prime} F\left(t^{\prime}\right) d\left(\boldsymbol{\kappa}_{t^{\prime}}\right) \\
& \times \int_{-\infty}^{t^{\prime}} d t^{\prime \prime} F\left(t^{\prime \prime}\right) d^{*}\left(\boldsymbol{\kappa}_{t^{\prime \prime}}\right) e^{-i S\left(\mathbf{k}, t^{\prime \prime}, t^{\prime}\right)-\left(t^{\prime}-t^{\prime \prime}\right) / T_{2}}+\text { c.c. },
\end{aligned}
$$

$$
\begin{aligned}
\mathbf{j}_{\mathrm{er}}(\omega)= & \omega \int_{\mathrm{BZ}} d^{3} \mathbf{k} \mathbf{d}(\mathbf{k}) \int_{-\infty}^{\infty} d t e^{-i \omega t} \int_{-\infty}^{t} d t^{\prime} F\left(t^{\prime}\right) d^{*}\left(\boldsymbol{\kappa}_{t^{\prime}}\right) \\
& \times e^{-i S\left(\mathbf{k}, t^{\prime}, t\right)-\left(t-t^{\prime}\right) / T_{2}}+\text { c.c., }
\end{aligned}
$$

where $\boldsymbol{\kappa}_{t^{\prime}}=\mathbf{k}+\mathbf{A}\left(t^{\prime}\right)-\mathbf{A}(t)$. Further, we have transformed the crystal momentum back to the initial frame $\mathbf{k}=\mathbf{K}+\mathbf{A}(t)$, and $S\left(\mathbf{k}, t^{\prime}, t\right)=\int_{t^{\prime}}^{t} \varepsilon_{\mathrm{g}}\left(\boldsymbol{\kappa}_{\tau}\right) d \tau$. Note that deriving Eqs. (4) directly from the Schrödinger equation would yield the same result except for the dephasing term. For the range of fields $F_{0} \leq 0.008$, explored in experiments 
[10], the harmonic spectra obtained from Eqs. (4) and by using the full two-band equations (1) are practically identical.

We focus first on understanding the physics driving interband HHG, which turns out to be the dominant process in the numerical results below. The mechanism driving intraband HHG is only briefly touched upon. Following the semiclassical method developed for atomic HHG [19], saddle point integration with regard to the three integrals $d^{3} \mathbf{k}, d t^{\prime}, d t$ in Eq. (4) gives three saddle point conditions

$$
\begin{aligned}
\nabla_{\mathbf{k}} S & =\Delta \mathbf{x}_{c}-\Delta \mathbf{x}_{v}=0, \\
\frac{d S}{d t^{\prime}} & =\varepsilon_{\mathrm{g}}\left[\mathbf{k}-\mathbf{A}(t)+\mathbf{A}\left(t^{\prime}\right)\right]=0, \\
\frac{d S}{d t} & =\varepsilon_{\mathrm{g}}(\mathbf{k})=\omega .
\end{aligned}
$$

A complete evaluation of the saddle point integrals can be done using the methods employed in Refs. [17,19,20]. Here, we focus on the physical picture revealed by the saddle point equations. In Eq. (5a), $\Delta \mathbf{x}_{m}=\int_{t^{\prime}}^{t} \mathbf{v}_{m} d t^{\prime \prime}=$ $\mathbf{x}_{m}(t)-\mathbf{x}_{m}\left(t^{\prime}\right)$ is the distance propagated by the electron (hole) in the conduction (valence) band ( $m=c, v)$ between the time of birth $t^{\prime}$ and the time of observation $t$; the velocity is $\mathbf{v}_{m}=\nabla_{\mathbf{k}} E_{m}$. The first equation states that HHG takes place only when electrons and holes reencounter each other, after having been accelerated and separated by the laser field after their birth through ionization. In contrast to HHG in gases, where the parent ion is too heavy to move, crystal hole dynamics is relevant, as hole and electron masses are comparable. Similar to HHG in atoms, Eq. (5b) states that electrons are born with zero momentum at time $t^{\prime}$ [19], $\mathbf{k}=\mathbf{A}\left(t=t^{\prime}\right)-\mathbf{A}\left(t^{\prime}\right)=0$. The finite band gap energy results in a complex birth time, which is responsible for tunnel ionization. Finally, Eq. (5c) represents conservation of energy: the electron-hole pair recombines and emits a photon $\omega$ with energy equal to the band gap at the momentum of recombination $\mathbf{k}=\mathbf{A}(t)-\mathbf{A}\left(t^{\prime}\right)$. As a result, the cutoff for interband HHG in a two-band model is limited to the maximum band gap. Higher harmonics are possible, but require excitation to a higher band.

Intraband HHG is driven by two mechanisms. First, Bloch oscillations are due to the anharmonic part of the band structure resulting in a nonlinear band velocity $v_{m}\left(\mathbf{k}=\mathbf{A}(t)-\mathbf{A}\left(t^{\prime}\right)\right)$ and therewith, a nonlinear current [15-17]. The second contribution has been ignored so far. The intraband current also contains a recollisionlike contribution that depends on the polarization buildup between electrons and holes due to the exponential term containing $S$ in Eq. (4a). Comparison of the two contributions is difficult, as they are closely intertwined.

Our analysis of HHG starts with a comparison of interband (blue) and intraband (red) HHG for dephasing times $T_{2}=\infty, T_{0} / 2, T_{0} / 4$ [Figs. $\left.1(\mathrm{a})-1(\mathrm{c})\right] ; T_{0}=2 \pi / \omega_{0}$ is the laser oscillation period. Nonperturbative HHG takes place above the dashed line $(N=9)$, indicating the minimum band gap at the center of the Brillouin zone. Both contributions show a plateau in the nonperturbative regime, whereas in experiments the harmonic yield drops with increasing harmonic order. This difference is due to the neglect of propagation effects, in particular reabsorption of harmonic photons, which increases with growing harmonic orders. For all dephasing times in Fig. 1 the interband current dominates the intraband contribution in the plateau; in the perturbative regime of HHG, below the dashed line, the two contributions become comparable with intraband HHG being slightly stronger. The calculation was repeated for a range of field strengths used in experiments [10]. Interband HHG is always dominant in the plateau region by at least 2 orders of magnitude.

Surprisingly, both spectra in Fig. 1(a) are very noisy and do not show a clear odd harmonics structure; only close to the cutoff can odd harmonics be seen. The lowest odd harmonic still visible is in agreement with the return energy above which higher returns cannot occur, as obtained from a solution of Eqs. (5). For harmonics below this energy interference of multiple returns washes out the odd harmonic structure. In Figs. 1(b) and 1(c) the spectra have been plotted for $T_{2}=T_{0} / 2, T_{0} / 4$. The odd harmonic spectrum starts to manifest for dephasing times of the order of a half cycle, see Fig. 1(b), as higher returns are suppressed. For even shorter dephasing times in Fig. 1(c), the signal to background ratio is further improved and a clean harmonic spectrum emerges.

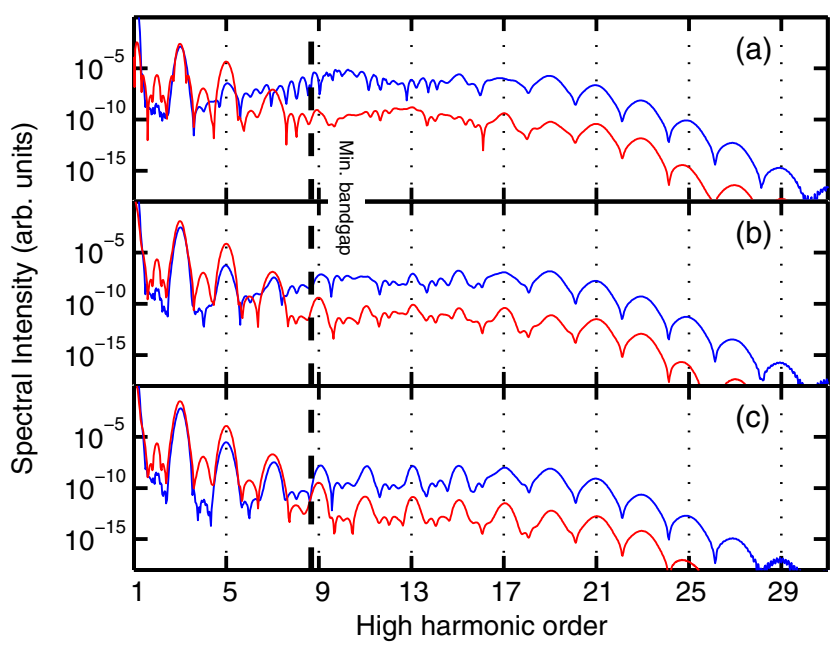

FIG. 1 (color online). Harmonic spectrum from interband $\left(\left|\mathrm{FT}\left\{\mathrm{j}_{\mathrm{er}}\right\}\right|^{2}\right.$, blue line $)$ and intraband $\left(\left|\mathrm{FT}\left\{\mathrm{j}_{\mathrm{ra}}\right\}\right|^{2}\right.$, red line $)$ currents for field strength $F_{0}=0.003$; we use a temporal Gaussian envelope with a FWHM equal to 10 cycles and cosine carrier with frequency $\omega_{0}=0.014$, corresponding to a laser period $T_{0}=2 \pi / \omega_{0}=10.9 \mathrm{fs}$; (a),(b),(c) show dephasing times of $T_{2}=\infty, T_{0} / 2, T_{0} / 4$, respectively. The dashed black vertical lines represent the (minimum) band gap at the center of the Brillouin zone. 
In atomic $\mathrm{HHG}$, propagation effects introducing a phase mismatch between the fundamental laser frequency and its harmonics can also contribute to attenuating second and higher returns [32]. This is not the case in solids, as absorption dominates phase mismatch for above band gap plateau harmonics [18], leaving dephasing as the only mechanism for obtaining a clean harmonic spectrum in agreement with experiments; this has important ramifications.

The dephasing time for which numerical and experimental peak-to-valley ratios of harmonics become comparable gives an upper limit of $T_{2}$. We find that for $T_{2} \approx 4 \mathrm{fs}$ the difference between harmonic peaks and valleys is around 1 to 1.5 orders of magnitude, comparable to what was found in experiments [10]. Typical dephasing times in semiconductors were found to be in the 15-50 fs range by photon echo experiments [33,34]; however, in these experiments only low energy electrons around the $\Gamma$ point are probed. Our analysis indicates that, in intense laser fields, dephasing can become even more efficient due to a population of the high crystal momentum (k) states in the Brillouin zone. An estimate of dephasing times at higher electron energies can be obtained from electron diffraction experiments. The measured scattering length for a $6 \mathrm{eV}$ electron is generally on the order of $100 \AA$, corresponding to a dephasing time of about 5 fs [35]. This estimate supports our findings.

In Fig. 2, we investigate how the interband (full lines) and intraband (dashed lines) contributions scale with laser

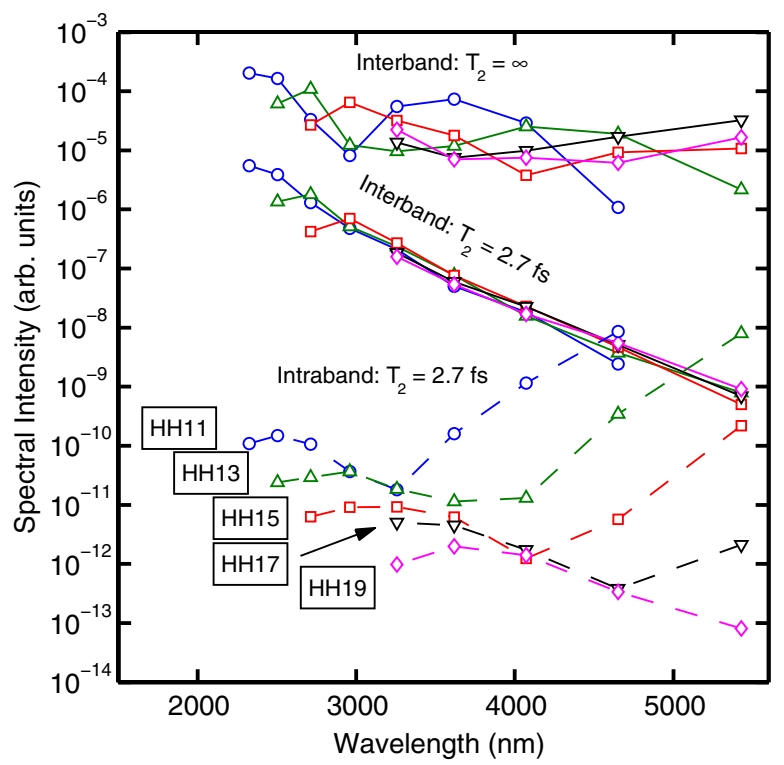

FIG. 2 (color online). Harmonic intensity versus laser wavelength $\lambda_{0}$ for various High-Harmonics (HH) orders; laser parameters are as in Fig. 1; circles, triangles pointed upward, squares, triangles pointed downward, and diamonds refer to $\mathrm{HH}$ orders 11 , $13,15,17$, and 19, respectively. The solid lines show the interband contributions for $T_{2}=2.7 \mathrm{fs}\left(T_{0} / 4\right.$ for $\left.\lambda_{0}=3.25 \mu \mathrm{m}\right)$ and $T_{2}=\infty$. The dashed lines (HH order numbered) show intraband contributions for $T_{2}=2.7 \mathrm{fs}$. wavelength $\lambda_{0}$ for $T_{2}=2.7 \mathrm{fs}$. When the wavelength is reduced, at some point a given harmonic will move beyond the plateau into the cutoff region and the yield will drop. On the other hand, for increasing wavelength a given harmonic order will drop below the band gap and move from the nonperturbative into the perturbative realm. We have plotted each harmonic only between these two limitsas long as it stays in the plateau region.

The interband contribution drops exponentially with increasing wavelength. This can be attributed mostly to the dephasing term in Eq. (4b); the harmonic yield drops by 4 orders of magnitude over the displayed wavelength range for $T_{2}=2.7$ fs (full lines). By comparison, interband HHG for $T_{2}=\infty$ (full lines) drops slowly and fluctuates within an order of magnitude. This behavior comes from an increase in the excursion time $t-t^{\prime}$ with growing $\lambda$. On the one hand, quantum spreading of 3D electron and hole wave packets is enhanced, which reduces recollision probability. On the other hand, phase and interference between multiple returns is modified resulting in the observed fluctuations. Finally, the wavelength dependence of the intraband yield is more complex. It most likely results from a mixing of the two different mechanisms identified above and can even increase for longer wavelengths.

For the chosen dephasing time, interband HHG clearly dominates over the range of nonperturbative HHG; some harmonics become comparable at the long wavelength end where the harmonic energy is comparable to the band gap. The calculations have been repeated for field strengths between $0.002 \leq F_{0} \leq 0.006$ and they show a very similar picture. For shorter dephasing times, intraband HHG gains more weight and the points where interband and intraband HHG intersect move slowly to shorter wavelengths; however, down to $T_{2} \approx 1 \mathrm{fs}$, interband HHG remains dominant over most of the displayed wavelength regime.

Dephasing times below a femtosecond are unrealistically short and therefore our calculations indicate the dominance of interband HHG in the nonperturbative part of the high harmonics spectrum over most of the investigated mid-ir range. However, as our model relies on approximations, this must ultimately be settled by experiment. Figure 2 reveals one suggestion of how this can be done. The opposite gradients of interband and intraband yield at longer wavelengths present a strong signature for experimentally discerning the two mechanisms.

Finally, we find that interband and intraband HHG show the same dependence on elliptical polarization and cannot be distinguished by ellipticity. The ellipticity dependence of HHG in solids is weaker than in gases. At an ellipticity of 0.25 , the harmonic intensity drops less than 1 order of magnitude as compared to linear polarization, whereas atomic HHG is already suppressed at lower ellipticities. However, we find a somewhat stronger drop of $\approx 2-3$ orders of magnitude than the factor of 5 measured in experiment for an ellipticity of 0.5 [10]. The difference might come 
from the neglect of electron-hole attraction, which weakens the effect of ellipticity.

In conclusion, the results presented in this work suggest that it will be possible to apply the methods of gas-phase high-harmonic spectroscopy to solids. For instance, we note that the spectral phase of the high-harmonics is determined by the $k$-dependent band gap. By perturbing the generation process with a two color experiment-just like in atomic HHG [36] - it is possible to measure this phase, from which ultimately the band structure of the crystal can be reconstructed. To date this has been possible only with angle resolved photoemission experiments. One can also envision a new class of solid state devices based on the methods developed for atomic gas recollision physics, but technically much easier to implement [37]. One example is the direct measurement of petahertz oscillations of a laser field [38], which was recently demonstrated with atomic HHG.

*giulio.vampa@nrc.ca

†cmcdo059@uottawa.ca

[1] P. B. Corkum, Phys. Rev. Lett. 71, 1994 (1993).

[2] P. B. Corkum and F. Krausz, Nat. Phys. 3, 381 (2007).

[3] J. Itatani, J. Levesque, D. Zeidler, H. Niikura, H. Pépin, J. C. Kieffer, P. B. Corkum, and D. M. Villeneuve, Nature (London) 432, 867 (2004).

[4] H. J. Wörner et al., Science 334, 208 (2011).

[5] X. Liu, D. Du, and G. Mourou, IEEE J. Quantum Electron. 33, 1706 (1997); D. Du, X. Liu, G. Korn, J. Squier, and G. Mourou, Appl. Phys. Lett. 64, 3071 (1994); B. C. Stuart, M. D. Feit, S. Herman, A. M. Rubenchik, B. W. Shore, and M. D. Perry, Phys. Rev. B 53, 1749 (1996).

[6] K. M. Davis, K. Miura, N. Sugimoto, and K. Hirao, Opt. Lett. 21, 1729 (1996).

[7] M. Schultze et al., Nature (London) 493, 75 (2013).

[8] A. Schiffrin et al., Nature (London) 493, 70 (2012).

[9] M. Krüger, M. Schenk, and P. Hommelhoff, Nature (London) 475, 78 (2011).

[10] S. Ghimire, A. D. DiChiara, E. Sistrunk, P. Agostini, L. F. DiMauro, and D. A. Reis, Nat. Phys. 7, 138 (2011).

[11] S. Ghimire, A. D. DiChiara, E. Sistrunk, U. B. Szafruga, P. Agostini, L. F. DiMauro, and D. A. Reis, Phys. Rev. Lett. 107, 167407 (2011).

[12] B. Zaks, R. B. Liu, and M. S. Sherwin, Nature (London) 483, 580 (2012).

[13] O. Schubert et al., Nat. Photonics 8, 119 (2014).

[14] D. Golde, T. Meier, and S. W. Koch, Phys. Rev. B 77, 075330 (2008).
[15] A. F. Kemper, B. Moritz, J. K. Freericks, and T. P. Deveraux, New J. Phys. 15, 023003 (2013).

[16] K. A. Pronin, A. D. Bandrauk, and A. A. Ovchinnikov, Phys. Rev. B 50, 3473 (1994).

[17] P. G. Hawkins and M. Yu. Ivanov, Phys. Rev. A 87, 063842 (2013).

[18] S. Ghimire, A. D. DiChiara, E. Sistrunk, G. Ndabashimiye, U. B. Szafruga, A. Mohammad, P. Agostini, L. F. DiMauro, and D. A. Reis, Phys. Rev. A 85, 043836 (2012).

[19] M. Lewenstein, P. Balcou, M. Y. Ivanov, A. L'Huillier, and P. B. Corkum, Phys. Rev. A 49, 2117 (1994).

[20] L. Keldysh, Sov. Phys. JETP 20, 1307 (1965).

[21] M. Goano, F. Bertazzi, M. Penna, and E. Bellotti, J. Appl. Phys. 102, 083709 (2007).

[22] See Supplemental Material at http://link.aps.org/ supplemental/10.1103/PhysRevLett.113.073901, which includes Refs. [23-30], for the derivation of the two-band equations and of the transition dipole moments.

[23] J. B. Krieger and G. J. Iafrate, Phys. Rev. B 33, 5494 (1986).

[24] J. B. Krieger and G. J. Iafrate, Phys. Rev. B 35, 9644 (1987).

[25] E. I. Blount, Solid State Phys. 13, 305 (1962).

[26] K. S. Virk and J. E. Sipe, Phys. Rev. B 76, 035213 (2007).

[27] I. Vurgaftman, J. R. Meyer, and L. R. Ram-Mohan, J. Appl. Phys. 89, 5815 (2001).

[28] Ü. Özgür, Ya. I. Alivov, C. Liu, A. Teke, M. A. Reshchikov, S. Doğan, V. Avrutin, S.-J. Cho, and H. Morkoç, J. Appl. Phys. 98, 041301 (2005).

[29] A. Schleife, F. Fuchs, C. Rödl, J. Furthmüller, and F. Bechstedt, Phys. Status Solidi B 246, 2150 (2009).

[30] Q. Yan, P. Rinke, M. Winkelnkemper, A. Qteish, D. Bimberg, M. Scheffler, and C. G. Van de Walle, Appl. Phys. Lett. 101, 152105 (2012).

[31] H. Haug and S. W. Koch, Quantum Theory of the Optical and Electronic Properties of Semiconductors (World Scientific, Singapore, 2004).

[32] Ph. Balcou, P. Salieres, A. L'Huillier, and M. Lewenstein, Phys. Rev. A 55, 3204 (1997).

[33] P. C. Becker, H. L. Fragnito, C. H. Brito Cruz, R. L. Fork, J. E. Cunningham, J. E. Henry, and C. V. Shank, Phys. Rev. Lett. 61, 1647 (1988).

[34] M. T. Portella, J.-Y. Bigot, R. W. Schoenlein, J. E. Cunningham, and C. V. Shank, Appl. Phys. Lett. 60, 2123 (1992).

[35] S. Hüfner, Photoelectron Spectroscopy: Principles and Applications (Springer, New York, 2003).

[36] N. Dudovich, O. Smirnova, J. Levesque, Y. Mairesse, M. Yu. Ivanov, D. M. Villeneuve, and P. B. Corkum, Nat. Phys. 2, 781 (2006).

[37] F. Krausz and M. I. Stockman, Nat. Photonics 8, 205 (2014).

[38] K. T. Kim, C. Zhang, A. D. Shiner, B. E. Schmidt, F. Légaré, D. M. Villeneuve, and P. B. Corkum, Nat. Photonics 7, 958 (2013). 